\title{
Control of Interconnected Mechanical Systems
}

\author{
Asif Šabanović*, Nadira Šabanović*, Kouhei Ohnishi** \\ *Sabancı University, Orhanl, 34956 Istanbul, Turkey \\ (Tel: 216-483-9502; e-mail: asif@, sabanciuniv.edu).nadira@, sabanciuniv.edu) \\ ** Keio University, Department of System Design, Yokohama, Japan,:
}

\begin{abstract}
In this paper control systems design approach, based on siding mode methods, that allows maintain some functional relation - like bilateral or multilateral systems, establishment of virtual relation among mobile robots or control of haptic systems - is presented. It is shown that all basic motion control problems - trajectory tracking, force control, hybrid position/force control scheme and the impedance control for the interacting systems- can be treated in the same way while avoiding the structural change of the controller and guarantying stable behavior of the system In order to show applicability of the proposed techniques simulation and experimental results for high precision systems in microsystems assembly tasks are presented..
\end{abstract}

\section{INTRODUCTION}

Motion control systems such as robots, vehicles and so on are expected to be applied in unstructured environment where presence of humans is natural. In many cases such systems are acting as "agents" between skilled human operator and environment (surgery, microparts handling, teleoperation, etc.), thus design of control should encompass wide range of very demanding tasks. In this paper a proposal for a new design framework for fully actuated mechanical system will be presented. The framework is based on the possibility to represent complex motion as a collection of the tasks specific functional relation between the system's state - that may be implemented concurrently or in certain time succession and than the design of the decentralized robust control - in particular sliding mode approach - to maintain these functional relations. Such an approach leads to more natural interpretation of the system tasks, simpler controller design and easier establishment of the systems hierarchy. The approach seems naturally encompasses the control of motion systems in interaction and it allows application to bilateral control, multilateral control, mobile robots cooperative work etc. The possibility to enforce certain functional relations between coordinates of one or more motion systems represents a basis of the proposed algorithm. It will be shown that all basic motion control problems can be treated in the same way while avoiding the structural change of the controller and guarantying stable behavior of the system. This framework can be naturally extended to the control of mechanical systems in interaction, like bilateral or multilateral control.

The decentralized control is well established approach in motion control systems. There are many applications of decentralized control, with concepts such as subsumption architecture, multi-agent system, cell structure Although design methods for decentralized control systems are interesting as concepts a simple framework in view of controller design is desired to cope with complexity of motion systems in interaction. In Tsuji at al., 2005 a framework of controller design based on functionality is discussed, in Onal and Sabanovic (2005) a bilateral control using sliding mode control applying functionality has been implemented. In Strassberg et al. (1992) basic approach in control of bilateral system widely used in literature is based on the design of the controllers for the master and the slave side separately and than adding interacting terms in order to reach the transparency requirements. Control of interconnected motion systems (bilateral and multilateral) in the framework of the acceleration control is discussed in Katsura and Ohishi (2007). In addition the so-called framework of functional control is discussed in Tsuji et al. (2006). All these works are based on the linearization of the individual systems by introducing the disturbance compensation and then applying acceleration control. This framework is shown to be very powerful and it allows generalization of the motion control systems design.

The application of SMC in motion control systems range from control of power converters, electrical machines, robotic manipulators, mobile robots, PZT based actuators etc. The most salient feature of the SMC is a possibility to constrain system motion on the selected manifold in the state space thus this framework seems natural candidate for the task we are pursuing in this paper - namely maintaining selected functional relation between systems. We will show that SMC framework can be interpreted as a way of implementing the acceleration control. In discrete-time this control that enforces sliding mode is continuous in a sense of the discrete-time systems and the resulting inter-sampling motion for systems with smooth disturbances is constrained to the $o\left(T^{2}\right)$ vicinity of the sliding manifold Utkin at al. (1999).

The organization of the paper is as follows. In Section 2 the problem formulation and general solution are discussed for $n$ degrees of freedom (DOF) fully actuated mechanical system with and/or without motion modification due to interaction 
with environment. In Section 3 the problems related to the modification of the systems motion due to the contact with environment are discussed and a possible solution is proposed. In Section 4 an extension of the solution presented in Section 3 is applied to motion control systems in interaction. In Section 5 the examples are shown in order to demonstrate applicability of the proposed framework..

\section{FULLY ACTUATED MECHANICAL SYSTEMS}

\subsection{Control Problem Formulation}

For fully actuated mechanical system $S$ mathematical model may be found in the following form

$$
\begin{aligned}
& S: \mathbf{M}(\mathbf{q}) \ddot{\mathbf{q}}+\mathbf{N}(\mathbf{q}, \dot{\mathbf{q}})=\mathbf{F}-\mathbf{F}_{\text {ext }}\left(\mathbf{q}, \mathbf{q}_{e}\right) ; \\
& \mathbf{F}_{\text {ext }}\left(\mathbf{q}, \mathbf{q}_{e}\right)=\left\{\begin{array}{cc}
\mathbf{g}_{i e}\left(\mathbf{q}, \mathbf{q}_{e}\right) & \text { with interactiont } \\
0 & \text { without interactiont }
\end{array}\right.
\end{aligned}
$$

where $\mathbf{q} \in \Re^{n}$ stands for vector of generalized positions, $\dot{\mathbf{q}} \in \mathfrak{R}^{n}$ stands for vector of generalized velocities, $\mathbf{M}(\mathbf{q}) \in \Re^{n \times n}$ is the generalized positive definite inertia matrix with bounded parameters hence $M^{-} \leq\|\mathbf{M}(\mathbf{q})\| \leq M^{+}$, $\mathbf{N}(\mathbf{q}, \dot{\mathbf{q}}) \in \Re^{n \times 1}$ represents vector of coupling forces including gravity and friction and is bounded by $\|\mathbf{N}(\mathbf{q}, \dot{\mathbf{q}})\| \leq N^{+}$, $\mathbf{F} \in \mathfrak{R}^{n \times 1}$ with $\|\mathbf{F}\| \leq F_{0}$ is vector of generalized input forces, $\mathbf{F}_{e x t} \in \mathfrak{R}^{n x 1}$ with $\left\|\mathbf{F}_{e x t}\right\| \leq F_{e x t}$ is vector of interaction forces being zero when system $S$ is not interacting with environment or other system and $\mathbf{q}_{e} \in \mathfrak{R}^{l}$ stands for the vector of generalized positions of environment. $M^{-}, M^{+}$, $N^{+}, F_{0}$ and $F_{e x t}$ are known scalars. In system (1) vectors $\mathbf{F}_{e x t}$ and $\mathbf{N}(\mathbf{q}, \dot{\mathbf{q}})$ are assumed to satisfy matching conditions Drazenovic (1969). For the purpose of this work the environment is treated as another mechanical system and the interaction is represented by the mechanical force acting as a result of such an interaction. It is now obvious that such an external force can be treated as an additional input to the system (1) able to modify the system behavior in the same way as the control input does.

The configuration of the fully actuated mechanical system can be represented by a single valued vector function $\xi(\mathbf{q}, \dot{\mathbf{q}}) \in \mathfrak{R}^{n x 1}$ as a function of the state of the system defined by the vector of generalized positions and vector of the generalized velocities. The control tasks for the system (1) are usually formulated as selection of the generalized input such that: (i) system executes desired motion specified as trajectory tracking, (ii) system exerts a desired force while in the contact with environment and (iii) system reacts as a desired impedance on the external force input or in contact with environment. In literature these problem are generally treated separately and motion that requires transition from one to another task is treated in the framework of hybrid control. All of these tasks may be represented as selected functions of the system configuration $\xi(\mathbf{q}, \dot{\mathbf{q}}) \in \mathfrak{R}^{n x 1}$. For example if the system configuration is expressed as a linear combination of the position and the velocity vectors $\xi(\mathbf{q}, \dot{\mathbf{q}})=\mathbf{C q}+\mathbf{Q} \dot{\mathbf{q}}$ it can be interpreted as a combination of spring (stiffness coefficient $\mathbf{C} \in \mathfrak{R}^{n \times n}$ ) and the damper (damping coefficient $\mathbf{Q} \in \mathfrak{R}^{n x n}$ ) and the mechanical impedance can be easily expressed as $\vartheta(\mathbf{q}, \dot{\mathbf{q}}, \ddot{\mathbf{q}})=\xi(\mathbf{q}, \dot{\mathbf{q}})+\boldsymbol{\Omega} \dot{\xi}(\mathbf{q}, \dot{\mathbf{q}})$ where $\boldsymbol{\Omega} \in \mathfrak{R}^{n x n}$ is full rank matrix. It is obvious that by changing the configuration of the system one can realize the change of the behavior of mechanical system in the case that is assumed isolated or having some interaction with environment. It is obvious that the definition of the system's (1) behavior in terms of the configuration is still open and it will be discussed later.

Assuming that the task of the system (1) can be described by a single valued vector function $\xi(\mathbf{q}, \dot{\mathbf{q}}) \in \mathfrak{R}^{n x 1}$ then the motion control of such a system can be formulated as the requirement to maintain the functional relation $\boldsymbol{\sigma}\left(\xi(\mathbf{q}, \dot{\mathbf{q}}), \xi^{\text {ref }}(t)\right)=\mathbf{0}, \boldsymbol{\sigma} \in \mathfrak{R}^{n \times 1} \quad$ between the actual configuration and the desired configuration $\xi^{\text {ref }}(t) \in \mathfrak{R}^{n \times 1}$ of the system (1). In the system's state space this requirement can be interpreted as enforcing the state of the system to stay in the manifold

$$
\begin{aligned}
& S_{q}=\left\{\mathbf{q}, \dot{\mathbf{q}}: \boldsymbol{\sigma}\left(\xi(\mathbf{q}, \dot{\mathbf{q}}), \xi^{r e f}\right)=\mathbf{0}\right\}, \\
& \boldsymbol{\sigma}, \xi, \xi^{r e f} \in \Re^{n x 1} ; \boldsymbol{\sigma}=\left[\sigma_{1}, \sigma_{2}, \ldots ., \sigma_{n}\right]^{T}
\end{aligned}
$$

where $\boldsymbol{\sigma} \in \mathfrak{R}^{n \times 1}$ is the linear of nonlinear single-valued vector function to be determined depending on the task of the overall system and the control system technical specification; the $\xi^{\text {ref }}(t) \in \Re^{n x 1}$ stands for the reference configuration of the system and is assumed to be smooth bounded function with a continuous first order time derivative. Requirement (2) is very simple - the configuration of the system should track reference configuration or in other word solution $\boldsymbol{\sigma}\left(\xi(\mathbf{q}, \dot{\mathbf{q}}), \xi^{\text {ref }}(t)\right)=\mathbf{0}$ must be stable on the trajectories of the system (1). With such a formulation the controller design is related to ensuring of the stability of solution $\boldsymbol{\sigma}\left(\xi(\mathbf{q}, \dot{\mathbf{q}}), \xi^{\text {ref }}(t)\right)=\mathbf{0}$. The control should be selected such that system state is enforced to satisfy functional relations specified by the manifold (2). The question of the definition of the operational tasks of system (1) in the terms of the system configuration and the admissible structure of the desired functional relation is still open and will require careful examination in order to complete overall control design. This problem will be addressed later in the paper.

Assume that controller is successfully enforcing the state of the system to satisfy the selected functional relation $\boldsymbol{\sigma}\left(\xi(\mathbf{q}, \dot{\mathbf{q}}), \xi^{\text {ref }}(t)\right)=\mathbf{0}$. By applying ideas proven in the framework of the sliding mode control $[* *]$ one can then claim that the state velocity vector must be orthogonal to the gradient of the manifold (2) such that the scalar product of the gradient vector and the velocity vector is zero for all 
configurations in manifold (2) thus $\operatorname{grad}\left(\boldsymbol{\sigma}\left(\xi(\mathbf{q}, \dot{\mathbf{q}}), \boldsymbol{\xi}^{r e f}(t)\right)\right) \cdot \mathbf{v}_{0}(\mathbf{q}, \dot{\mathbf{q}})=\mathbf{0}$. This relation is not so easy for application and we will be using so-called equivalent control method $[* *]$ in order to determine the velocity vector (and thus the dynamics of the system (1)) in manifold (2). This method is taking as the control input to system (1) the solution of the algebraic equation $\left.\frac{d \boldsymbol{\sigma}\left(\xi(\mathbf{q}, \dot{\mathbf{q}}), \xi^{r e f}(t)\right)}{d t}\right|_{F=F_{\text {eq }}}=\mathbf{0}$ on the trajectories of system (1).

The time derivative of the function $\boldsymbol{\sigma}$ can be expressed as $\frac{d}{d t} \boldsymbol{\sigma}\left(\xi(\mathbf{q}, \dot{\mathbf{q}}), \xi^{r e f}(t)\right)=\left[\frac{\partial \boldsymbol{\sigma}}{\partial \xi} \frac{\partial \xi}{\partial \mathbf{q}}\right] \dot{\mathbf{q}}+\left[\frac{\partial \boldsymbol{\sigma}}{\partial \xi} \frac{\partial \xi}{\partial \dot{\mathbf{q}}}\right] \ddot{\mathbf{q}}+\left[\frac{\partial \boldsymbol{\sigma}}{\partial \xi^{r e f}}\right] \dot{\xi}^{r e f}$. For the sake of simplicity assume that matrices $\mathbf{C}=\left[\frac{\partial \boldsymbol{\sigma}}{\partial \xi} \frac{\partial \xi}{\partial \mathbf{q}}\right] \in \Re^{n x n}, \mathbf{Q}=\left[\frac{\partial \boldsymbol{\sigma}}{\partial \xi} \frac{\partial \xi}{\partial \dot{\mathbf{q}}}\right] \in \Re^{n x n}, \quad \mathbf{H}=\left[\frac{\partial \boldsymbol{\sigma}}{\partial \xi^{r e f}}\right] \in \Re^{n x n} \quad$ have full rank for $\forall(\mathbf{q}, \dot{\mathbf{q}}) \in S_{q}$ and consequently the relation $\left(\mathbf{Q} \mathbf{M}^{-1}\right)^{-1}=\mathbf{M} \mathbf{Q}^{-1}$ is true due to the properties of the inertia matrix. The equivalent control for system (1) in manifold (2) can be obtined by inserting $\mathbf{M}(\mathbf{q}) \ddot{\mathbf{q}}+\mathbf{N}(\mathbf{q}, \dot{\mathbf{q}})=\mathbf{F}-\mathbf{F}_{e x t}\left(\mathbf{q}, \mathbf{q}_{e}\right)$ into $\mathbf{C} \dot{\mathbf{q}}+\mathbf{Q} \ddot{\mathbf{q}}+\mathbf{H} \dot{\xi}^{r e f}=\mathbf{0}$ :

$$
\mathbf{F}_{e q}=\left(\mathbf{F}_{e x t}\left(\mathbf{q}, \mathbf{q}_{e}\right)+\mathbf{N}(\mathbf{q}, \dot{\mathbf{q}})\right)-\left(\mathbf{Q} \mathbf{M}^{-1}\right)^{-1}\left(\mathbf{H} \dot{\xi}^{r e f}(t)+\mathbf{C} \dot{\mathbf{q}}\right)
$$

Note that equivalent control is smooth as long as external forces and the first time derivative of the reference configuration are smooth functions. The expression (3) shows a very important fact - in order to maintain motion of the system (1) in the manifold (2) the control must compensate the internal interaction forces $(\mathbf{N}(\mathbf{q}, \dot{\mathbf{q}}))$ and external interaction forces $\left(\mathbf{F}_{\text {ext }}\left(\mathbf{q}, \mathbf{q}_{e}\right)\right)$ or in other words controller must compensate all disturbances acting on the system. This can be achieved since we assumed that $\mathbf{N}(\mathbf{q}, \dot{\mathbf{q}})$ and $\mathbf{F}_{e x t}\left(\mathbf{q}, \mathbf{q}_{e}\right)$ satisfy matching conditions. This result is consistent with the disturbance compensation method (**) widely used in the motion control systems design.

By inserting (3) into (1) equations of motion of fully actuated mechanical system (1) in manifold (2) are obtained in the following form

$$
\begin{aligned}
& \mathbf{M} \ddot{\mathbf{q}}=-\left(\mathbf{Q} \mathbf{M}^{-1}\right)^{-1}\left(\mathbf{H} \dot{\xi}^{r e f}(t)+\mathbf{C} \dot{\mathbf{q}}\right)=\mathbf{M} \ddot{\mathbf{q}}^{d e s} \Rightarrow \ddot{\mathbf{q}}=\ddot{\mathbf{q}}^{d e s} \\
& \ddot{\mathbf{q}}^{d e s}=-\mathbf{Q}^{-1}\left(\mathbf{H} \dot{\xi}^{r e f}(t)+\mathbf{C} \dot{\mathbf{q}}\right)
\end{aligned}
$$

The resulting motion (4) is valid from for the time $t \geq t_{0}$ with $t_{0}$ being the moment the state of the system reaches manifold (2). This way (4) can be regarded as a motion with the initial condition $\boldsymbol{\sigma}\left(\xi(\mathbf{q}, \dot{\mathbf{q}}), \xi^{r e f}(t)\right)=\mathbf{0}$. Motion from the initial state until reaching the manifold (2) will be discussed later in the paper and control will be selected such that so called reaching phase is short comparing with overall control time. Thus the motion is equivalent to the acceleration control [25] with desired acceleration $\ddot{\mathbf{q}}^{\text {des }}=-\mathbf{Q}^{-1}\left(\dot{\xi}^{r e f}(t)+\mathbf{C} \dot{\mathbf{q}}\right)$. Due to the fact that closed loop behavior does not depend on the external interaction force the motion of the system will not be modified while in contact with environment. Closed loop motion will not depend on the interaction forces as long as the equivalent control is bounded $\left\|\mathbf{F}_{e q}\right\| \leq F_{0}$ as defined for the system input in the description of the system. If closed loop motion (4) should be modified due to the interaction with other systems or environment the desired acceleration $\ddot{\mathbf{q}}^{\text {des }}$ must depend on the interaction force. That can be achieved in many different ways. Obvious one it to make reference configuration function of the interaction force or to select control such that the desired part of the interaction force is not compensated by the controller. The first approach leads to modification of the reference in order to achieve desired behavior - what sometimes may be advantageous since it is kept outside of the main loop so it theoretically should not affect the stability of the system. The other approach is shifting the problem towards designing the observer that can provide information on the part of the interaction force that needs to be used to modify motion and in a sense leads to some sort of the decoupled design - controller design and the observer design.

\subsection{Selection of the control input}

The simplest and the most direct method to derive a control, which enforces the selected functional relation $\boldsymbol{\sigma}\left(\xi, \xi^{r e f}\right)=\mathbf{0}_{n x 1}$ is to enforce Lyapunov stability conditions in the manifold (2) on the trajectories of system (1). To guaranty stability of solution $\boldsymbol{\sigma}=\mathbf{0}_{n x 1}$ a Lyapunov function candidate may be selected as $v=\frac{1}{2} \boldsymbol{\sigma}^{T} \boldsymbol{\sigma}>0, v(0)=0$ and one has to design control that enforces the following structure of the time derivative $\dot{v}=\boldsymbol{\sigma}^{T} \dot{\boldsymbol{\sigma}}=-\boldsymbol{\sigma}^{T} \boldsymbol{\Psi}(\boldsymbol{\sigma})<0$ of the selected Lyapunov function. For example if $-\boldsymbol{\sigma}^{T} \boldsymbol{\Psi}(\boldsymbol{\sigma})=-\rho v^{\delta}<0$ the manifold (2) will be attractive and the stability conditions for solution $\boldsymbol{\sigma}\left(\xi, \xi^{r e f}\right)=\mathbf{0}_{n x 1}$ are satisfied. In order to guaranty the finite time convergence to the manifold (2) the coefficients $\rho, \delta$ should be selected as $\rho>0$ and $\frac{1}{2} \leq \delta<1$ [27] in order to guaranty finite reaching time and the stability. For $\delta=1$ we can say only that the convergence is not slower than exponential. In order to determine control input one should use the requirement for the Lyapunov function derivative $\dot{v}=\boldsymbol{\sigma}^{T} \dot{\boldsymbol{\sigma}}=-\boldsymbol{\sigma}^{T} \boldsymbol{\Psi}(\boldsymbol{\sigma})$. From here nontrivial solution can be obtained from $\dot{\boldsymbol{\sigma}}+\left.\boldsymbol{\Psi}(\boldsymbol{\sigma})\right|_{\sigma \neq 0}=0$ finding the following form

$$
\begin{aligned}
& \mathbf{F}=\mathbf{F}_{e q}-\left(\mathbf{Q} \mathbf{M}^{-1}\right)^{-1} \mathbf{\Psi}(\boldsymbol{\sigma})=\mathbf{F}_{e q}-\mathbf{M} \mathbf{Q}^{-1} \mathbf{\Psi}(\boldsymbol{\sigma}) \\
& \mathbf{F}_{e q}=\left(\mathbf{F}_{e x t}\left(\mathbf{q}, \mathbf{q}_{e}\right)+\mathbf{N}(\mathbf{q}, \dot{\mathbf{q}})\right)-\mathbf{M Q}^{-1}\left(\mathbf{H} \dot{\xi}^{r e f}(t)+\mathbf{C} \dot{\mathbf{q}}\right)
\end{aligned}
$$

For continuous-time systems function $\Psi(\boldsymbol{\sigma})$ is most often selected to satisfy $-\boldsymbol{\sigma}^{T} \boldsymbol{\Psi}(\boldsymbol{\sigma})=-\rho v^{1 / 2}$. The resulting control guaranties so called sliding mode motion in manifold (2). Being discontinuous such control input may cause chattering in mechanical systems. For real system chattering may be a 
problem and many possibilities to avoid or minimize chattering problem in mechanical systems are presented in literature Utkin at al., (1999).

In the real system control is bounded $\|\mathbf{F}\| \leq F_{0}$ so (5) should be modified to take this into account. The modification is simple, instead (5) the real control input should satisfy

$$
\mathbf{F}=\operatorname{sat}\left(\mathbf{F}_{e q}-\mathbf{M Q}^{-1} \boldsymbol{\Psi}(\boldsymbol{\sigma})\right)
$$

where $\operatorname{sat}(\bullet)$ stands for saturation function with bounds $\mathbf{F}_{b}=F_{0} \frac{\mathbf{F}}{\|\mathbf{F}\|}$. If the control takes values within available control resources $\|\mathbf{F}\|<F_{0}$ the stability conditions in manifold (2) are enforced. If the required control resources are $\|\mathbf{F}\| \geq F_{0}$ one can find the derivative of the function $\boldsymbol{\sigma}\left(\xi, \xi^{\text {ref }}\right)$ as $\dot{\boldsymbol{\sigma}}=\left(\mathbf{Q} \mathbf{M}^{-1}\right)\left(\mathbf{F}-\mathbf{F}_{e q}\right)=\left(\mathbf{Q} \mathbf{M}^{-1}\right)\left(F_{0} \frac{\mathbf{F}}{\|\mathbf{F}\|}-\mathbf{F}_{e q}\right)$. By inserting

and taking into account that in this region $\|\mathbf{F}\|=F_{0}$ one can find. $\dot{\boldsymbol{\sigma}}=-\left(1-\frac{F_{0}}{\|\mathbf{F}\|}\right) F_{0}-\frac{F_{0}}{\|\mathbf{F}\|} \boldsymbol{\Psi}(\boldsymbol{\sigma})$ hence $\boldsymbol{\sigma}$ decreases and after finite time region $\|\mathbf{F}\|<F_{0}$ is reached. This way it has been proven that the control (6) guaranties the attractiveness and the stability of $\boldsymbol{\sigma}\left(\xi, \xi^{r e f}\right)=\mathbf{0}_{n x 1}$. Selected control enforces $\dot{v}=\boldsymbol{\sigma}^{T} \dot{\boldsymbol{\sigma}}=-\boldsymbol{\sigma}^{T} \boldsymbol{\Psi}(\boldsymbol{\sigma})<0$ on the trajectories of the system (1).

The implementation of the control (6) is not so easy due to the fact that the disturbances should be known. If one applies disturbance observer than control can be modified to the form

$$
\mathbf{F}=\operatorname{sat}\left(\left(\hat{\mathbf{F}}_{e x t}+\hat{\mathbf{N}}\right)-\mathbf{M} \mathbf{Q}^{-1}\left(\mathbf{H} \dot{\xi}^{r e f}(t)+\mathbf{C} \dot{\mathbf{q}}+\mathbf{\Psi}(\boldsymbol{\sigma})\right)\right)
$$

Approximated control (7) enforces the relation $\dot{\boldsymbol{\sigma}}+\Psi(\boldsymbol{\Psi})=\mathbf{p}$ where $\mathbf{p}=\left(\mathbf{F}_{\text {ext }}+\mathbf{N}\right)-\left(\hat{\mathbf{F}}_{\text {ext }}+\hat{\mathbf{N}}\right)$. The consequence is that in such a system some error may appear and the additional measures should be taken to compensate for it. The structure (7) represents a generic acceleration controller enforcing the attractiveness and the stability of $\boldsymbol{\sigma}\left(\xi, \xi^{\text {ref }}\right)=\mathbf{0}$. We will be later showing that PD controller with disturbance feed forward is a particular case of (7).

In the discrete time with sampling interval " $T$ " implementation of control (6) requires evaluation of the equivalent control at the end of every sampling interval. The equivalent control is continuous function so one can resort of using its value at $t=(k-1) T$ instead of the exact value at $t=k T$. By evaluating $\dot{\boldsymbol{\sigma}}=\mathbf{Q} \mathbf{M}^{-1}\left(\mathbf{F}-\mathbf{F}_{e q}\right)$ at $t=(k-1) T$ it is easy to derive $\mathbf{F}_{e q}(k-1)=\left(\mathbf{F}(k-1)-\mathbf{M Q}^{-1} \dot{\boldsymbol{\sigma}}(k-1)\right)$. Approximation $\quad \dot{\boldsymbol{\sigma}}(k-1)=(\boldsymbol{\sigma}(k)-\boldsymbol{\sigma}(k-1)) / T$ leads to $\mathbf{F}_{e q}(k-1)=\left(\mathbf{F}(k-1)-\mathbf{M} \mathbf{Q}^{-1} T^{-1}(\boldsymbol{\sigma}(k)-\boldsymbol{\sigma}(k-1))\right) \quad$ with $\quad$ an approximation error of the $o\left(T^{2}\right)$ order. Now the approximated control input can be expressed as

$$
\begin{aligned}
& \mathbf{F}(k) \cong \operatorname{sat}\left(\mathbf{F}_{e q}(k-1)-\mathbf{M} \mathbf{Q}^{-1} \mathbf{\Psi}\left(\boldsymbol{\sigma}_{k}\right)\right) \\
& \mathbf{F}(k)=\operatorname{sat}(\underbrace{\left(\mathbf{F}(k-1)-\mathbf{M} \mathbf{Q}^{-1} T^{-1}(\boldsymbol{\sigma}(k)-\boldsymbol{\sigma}(k-1))\right)}_{\mathbf{F}_{e q}(k-1)}-\mathbf{M} \mathbf{Q}^{-1} \mathbf{\Psi}(\boldsymbol{\sigma}(k)))
\end{aligned}
$$

By inserting (8) into (1) one can evaluate system dynamics at $t=k T$ as

$\mathbf{M} \ddot{\mathbf{q}}(k)=\mathbf{F}_{e q}(k-1)-\mathbf{M} \mathbf{Q}^{-1} \boldsymbol{\Psi}(\boldsymbol{\sigma}(k))-\left(\mathbf{F}_{e x t}(k)+\mathbf{N}(\mathbf{q}(k), \dot{\mathbf{q}}(k))\right)$

The error introduced by this approximation of control can be estimated from the following relation

$$
\begin{aligned}
& \dot{\boldsymbol{\sigma}}(k)+\boldsymbol{\Psi}(\boldsymbol{\sigma}(k))=-\mathbf{Q} \mathbf{M}^{-1}\left(\mathbf{F}_{e q}(k)-\mathbf{F}_{e q}(k-1)\right) \\
& \mathbf{Q M}^{-1}\left(\mathbf{F}_{e q}(k)-\mathbf{F}_{e q}(k-1)\right) \cong o(T)
\end{aligned}
$$

Due to the continuity of the equivalent control the thickness of the boundary layer for the manifold (2) (intersampling change of the distance from the manifold) to which motion of the system will be confined to can be determined by evaluating $\boldsymbol{\sigma}(k T+\tau)-\boldsymbol{\sigma}(k T)=-\int_{k T}^{k T+\tau} \boldsymbol{\Psi}(\boldsymbol{\sigma}(t)) d t+o\left(T^{2}\right) . \quad$ If $\quad \boldsymbol{\Psi}(\boldsymbol{\sigma}(t)) \quad$ is proportional to $\boldsymbol{\sigma}(t)$ then the thickness of the boundary layer is $o\left(T^{2}\right)$ order. If relay control is applied it will result in motion with chattering within a boundary layer having thickness of the $o(T)$ order.

\section{SELECTION OF THE REFERENCE CONFIGURATION}

\subsection{Position and force control}

In literature behavior of a motion control system is mostly analyzed in three separate frameworks: (i) the trajectory tracking, (ii) the force control and (iii) impedance control. Due to the fact that in fully actuated systems interaction forces and system configuration cannot be set independently hybrid schemes had been developed to cope with position-force control tasks and the transitions from one to another [26]. In the proposed framework the solution of the above control problems require selection of the appropriate system configuration, the reference configuration and the functional constraint (2). As shown in Section 2. closed loop behavior of the system (1) in manifold (2) is described by the second order differential equation (4) and $\boldsymbol{\sigma}\left(\xi, \xi^{\text {ref }}\right)=\mathbf{0}$ (in ideal case) or $\boldsymbol{\sigma}\left(\xi, \xi^{r e f}\right)=o(T)$ (in discrete time system with equivalent control approximation) with transient dynamics described by $\dot{\boldsymbol{\sigma}}+\left.\boldsymbol{\Psi}(\boldsymbol{\sigma})\right|_{\sigma \neq 0}=0 . \quad$ Without loss of generality let assume that matrices $\mathbf{C}, \mathbf{Q}, \mathbf{H}$ are constant - thus that configuration of the system is a linear combination of the generalized position and velocity $\xi_{q}(t)=(\mathbf{Q} \dot{\mathbf{q}}+\mathbf{C q})$ and the functional relation (2) is also linear combination of the reference and actual configuration. Assume the trajectory tracking problem. By selecting $\xi_{q}^{r e f}(t)=\left(\mathbf{Q} \dot{\mathbf{q}}^{r e f}+\mathbf{C} \mathbf{q}^{r e f}\right)$ with manifold (2) $S_{q}=\left\{\mathbf{q}, \dot{\mathbf{q}}: \boldsymbol{\sigma}\left(\xi, \xi^{r e f}\right)=\xi^{r e f}-\xi(\mathbf{q}, \dot{\mathbf{q}})=\mathbf{0}\right\}$ the control (7) or (8) can be directly applied. If one selects 
$\Psi\left(\boldsymbol{\sigma}_{q}\right)=-\mathbf{D} \boldsymbol{\sigma}_{q} ; \mathbf{D}>0$ the equations of motion can be determined in the following form $\mathbf{Q}\left(\ddot{\mathbf{q}}-\ddot{\mathbf{q}}^{\text {ref }}\right)+(\mathbf{C}+\mathbf{D Q})\left(\dot{\mathbf{q}}-\dot{\mathbf{q}}^{\text {ref }}\right)+\mathbf{C D}\left(\mathbf{q}-\mathbf{q}^{\text {ref }}\right)=\mathbf{0}$. This result is the same as the one obtained by application of the disturbance observer and PD controller as discussed in [25]. This can be interpreted as a system with the mechanical impedance having mass $\mathbf{Q}$, damping $(\mathbf{C}+\mathbf{D Q})$ and spring coefficient $\mathbf{C D}$. If the matrix $\mathbf{D}$ is selected diagonal and its elements such that the transient $\dot{\boldsymbol{\sigma}}_{q}+\mathbf{D} \boldsymbol{\sigma}_{q}=\mathbf{0}$ is fast as compared to the sliding mode dynamics (defined by matrices $\mathbf{Q}$ and $\mathbf{C}$ ), after initial transient the resulting motion remains in the $\mathcal{E}$ - thick boundary layer of $\boldsymbol{\sigma}_{q}=\mathbf{0}$.

In the force control with the reference $\mathbf{F}^{\text {ref }}(t)$ the sliding mode manifold can be defined as $S_{F}=\left\{(\mathbf{q}, \dot{\mathbf{q}}): \mathbf{F}(\mathbf{q}, \dot{\mathbf{q}})-\mathbf{F}^{r e f}(t)=\boldsymbol{\sigma}_{F}=\mathbf{0}\right\}$. If measured force is modeled as $\mathbf{F}(\mathbf{q}, \dot{\mathbf{q}})=\mathbf{K}_{P} \Delta \mathbf{q}+\mathbf{K}_{D} \Delta \dot{\mathbf{q}}, \Delta \mathbf{q}=\mathbf{q}-\mathbf{q}_{e}, \mathbf{K}_{P}, \mathbf{K}_{D}$ are diagonal matrices of appropriate dimensions, then by defining the reference configuration as $\boldsymbol{\xi}_{F}^{r e f}(t)=\left(\mathbf{F}^{\text {ref }}(t)+\mathbf{K}_{P} \mathbf{q}_{e}+\mathbf{K}_{D} \dot{\mathbf{q}}_{e}\right)$ the manifold (2) becomes $S_{F}=\left\{(\mathbf{q}, \dot{\mathbf{q}}): \mathbf{K}_{P} \mathbf{q}+\mathbf{K}_{D} \dot{\mathbf{q}}-\boldsymbol{\xi}_{F}^{r e f}(t)=\boldsymbol{\sigma}_{F}=\mathbf{0}\right\}$. This manifold has the same form as the one derived for the trajectory tracking, thus the control input is as in (8), which is the same as for trajectory tracking with appropriate changes of the variables.

\subsection{Motion modification due to the systems interaction}

Assume two mechanical systems $S_{i}$ and $S_{j}$ as in (1) with reference configurations $\xi_{i q}^{r e f}(t)$ and $\xi_{j q}^{r e f}(t)$ respectively. Let the interaction forces between systems $S_{i}$ and $S_{j}$ be denoted as $\mathbf{g}_{i j}\left(\mathbf{q}_{i}, \mathbf{q}_{j}\right) \in \mathfrak{R}^{n \times 1}$, which becomes $\mathbf{g}_{i j}\left(\mathbf{q}_{i}, \mathbf{q}_{j}\right)=\mathbf{0}$ if systems are not in interaction. Assume that this force can be modeled as $\mathbf{g}_{i j}\left(\mathbf{q}_{i}, \mathbf{q}_{j}\right)=\mathbf{K}_{P i} \Delta \mathbf{q}+\mathbf{K}_{D i} \Delta \dot{\mathbf{q}}, \Delta \mathbf{q}=\mathbf{q}_{i}-\mathbf{q}_{j}$ and should be maintained at the desired value $\mathbf{g}_{i j}^{\text {ref }}(t)$ while the systems are in interaction. Assume that only system $S_{i}$ should is able to its configuration as result of the interaction in order to maintain the desired profile of the interaction force. This can be achieved by making reference configuration of system $S_{i}$ dependent on the interaction force $\mathbf{g}_{i j}\left(\mathbf{q}_{i}, \mathbf{q}_{j}\right)$ one can make motion of the system reacting on both the reference trajectory and the interaction force. The modification of the trajectory of the system $S_{i}$ could be selected (i) to be proportional with the interaction force (so-called compliant motion), (ii) to ensure that the interaction force tracks its reference (force tracking), and (iii) the combination of the cases (i) and (ii).

For all three cases the manifold has the following form

$S_{i q}=\left\{\mathbf{q}_{i}, \dot{\mathbf{q}}_{i}: \mathbf{C}_{i} \mathbf{q}_{i}+\mathbf{Q}_{i} \dot{\mathbf{q}}_{i}-\xi_{i q F}^{r e f}(t)=\boldsymbol{\sigma}_{i q F}=\mathbf{0}\right\}$
Depending on the specific task, the reference configuration $\xi_{q F}^{r e f}(t)$ can take one of the following forms

(i) $\boldsymbol{\xi}_{i q F}^{\text {ref }}(t)=\boldsymbol{\xi}_{i q}^{\text {ref }}(t)-\boldsymbol{\Gamma} \boldsymbol{g}_{i j}\left(\mathbf{q}_{i}, \mathbf{q}_{j}\right)$ or

(ii) $\boldsymbol{\xi}_{i q F}^{\text {ref }}(t)=\boldsymbol{\xi}_{i q}^{\text {ref }}(t)-\vartheta\left(\mathbf{g}_{i j}^{\text {ref }}(t), \mathbf{g}_{i j}\left(\mathbf{q}_{i}, \mathbf{q}_{j}\right)\right)$ or

(iii) $\boldsymbol{\xi}_{i q F}^{\text {ref }}(t)=\xi_{i q}^{\text {ref }}(t)-\left(\vartheta\left(\mathbf{g}_{i j}^{\text {ref }}(t), \mathbf{g}_{i j}\left(\mathbf{q}_{i}, \mathbf{q}_{j}\right)\right)+\Gamma \mathbf{g}_{i j}\left(\mathbf{q}_{i}, \mathbf{q}_{j}\right)\right)$

where the reference configuration for position tracking task is selected as $\xi_{i q}^{\text {ref }}=\mathbf{C}_{i} \mathbf{q}_{i}^{\text {ref }}+\mathbf{Q}_{i} \dot{\mathbf{q}}_{i}^{\text {ref }}$, matrix $\boldsymbol{\Gamma}$ is diagonal compliance matrix with elements different from zero in the directions in which compliance is to be maintained, and zero in the directions in which either contact force or trajectory tracking should be maintained. The output of the force tracking controller $\vartheta\left(\mathbf{g}_{i j}^{\text {ref }}, \mathbf{g}_{i j}\right)$ enforces sliding mode on the manifold $S_{i j F}=\left\{\left(\mathbf{q}_{i}, \dot{\mathbf{q}}_{i}\right): \mathbf{K}_{P i} \mathbf{q}_{i}+\mathbf{K}_{D i} \dot{\mathbf{q}}_{i}-\xi_{F}^{r e f}(t)=\sigma_{F i j}=\mathbf{0}\right\}$ with the reference configuration defied by $\boldsymbol{\xi}_{F}^{r e f}(t)=\left(\mathbf{g}_{i j}^{r e f}(t)+\mathbf{K}_{P i} \mathbf{q}_{j}+\mathbf{K}_{D i} \dot{\mathbf{q}}_{j}\right)$ and is determined as in (7) or (8) (with necessary changes of coordinates) when systems are in interaction and $\vartheta\left(\mathbf{g}_{i j}^{r e f}, \mathbf{g}_{i j}\right)=0$ when systems are not in interaction.

\subsection{Extension to the General Systems in Interactions}

Assume a set of $n$ single DOF motion systems each described by $\quad S_{i}: m_{i}\left(q_{i}\right) \ddot{q}_{i}+n_{i}\left(q_{i}, \dot{q}_{i}\right)=f_{i}-g_{\text {iext }} \quad i=1,2, \ldots, n$ interconnected in such a way so the motion of the overall system can be described by the following model

$$
S: \mathbf{M}(\mathbf{q}) \ddot{\mathbf{q}}+\mathbf{N}(\mathbf{q}, \dot{\mathbf{q}})=\mathbf{B F}-\mathbf{B g}_{i j}
$$

where $\quad \mathbf{q} \in \Re^{n}, \operatorname{rank} \mathbf{B}=\operatorname{rank} \mathbf{M}=n, \quad$ vectors $\quad \mathbf{N}$ and $\mathbf{B g}_{i j}$ satisfy matching conditions. Assume also that the required role $\boldsymbol{\Phi} \in \mathfrak{R}^{m}$ may be represented as a set of smooth linearly independent functions $\zeta_{1}(\mathbf{q}), \zeta_{2}(\mathbf{q}), \ldots, \zeta_{n}(\mathbf{q})$. . Consider a problem of designing control for system $S$ such that the role vector $\boldsymbol{\Phi}^{T}=\left[\begin{array}{lll}\zeta_{1}(\mathbf{q}) & \ldots & \zeta_{n}(\mathbf{q})\end{array}\right]$ tracks its smooth reference $\boldsymbol{\Phi}^{\text {ref }}$. By differentiating the first time derivative of the role vector $\dot{\mathbf{\Phi}}=\left\lfloor\frac{\partial \Phi}{\partial \mathbf{q}} \mid \dot{\mathbf{q}}=\mathbf{J}_{\Phi} \dot{\mathbf{q}}\right.$ by denoting the control vector as $\mathbf{F}_{\Phi}=\mathbf{B}_{\Phi} \mathbf{F}=\mathbf{J}_{\Phi} \mathbf{M}^{-1} \mathbf{B}$ and the disturbance vector as $\mathbf{d}_{\Phi}=\mathbf{J}_{\Phi} \mathbf{M}^{-1}\left(\mathbf{B g}_{i j}+\mathbf{N}\right)+\dot{\mathbf{J}}_{\Phi} \dot{\mathbf{q}}$ one can determine

$\ddot{\boldsymbol{\Phi}}=\mathbf{F}_{\Phi}-\mathbf{d}_{\Phi}$

From (11) one can select control $\mathbf{F}_{\Phi}=\mathbf{F}_{\Phi}^{*}$ such that the role vector tracks its reference and than, if $\left(\mathbf{B}_{\Phi}\right)^{-1}$ exists determine the original control input as $\mathbf{F}=\left(\mathbf{B}_{\Phi}\right)^{-1} \mathbf{F}_{\Phi}^{*}=\left(\mathbf{J}_{\Phi} \mathbf{M}^{-1} \mathbf{B}\right)^{-1} \mathbf{F}_{\Phi}^{*}$. In the general case one has to ensure the existence of the inverse for matrix $\mathbf{B}_{\Phi}$ by properly selecting matrix $\mathbf{J}_{\Phi}$. That defines the condition for selection of the structure of the function vector. Similar situation had been examined in so-called "function control" framework Tsuji at all (2006), There it was assumed that 
$\mathbf{B}=\mathbf{I}$ and $\mathbf{J}_{\Phi}$ was selected as a Hadamard matrix along with the compensation of the disturbances $\mathbf{g}_{i j}$ and $\mathbf{N}$ on the plant level. This way control design is greatly simplified and one deals with simple double integrator plants without disturbance.

The same problem can be treated in the above discussed framework by selecting the system configuration $\xi_{\Phi}(\boldsymbol{\Phi}, \dot{\boldsymbol{\Phi}})$ and the reference configuration $\xi_{\Phi}^{r e f}(t)$. Assume that the configuration is expressed as a linear combination of the function vector and its derivative $\xi(\Phi, \dot{\Phi})=\mathbf{C}_{\Phi} \boldsymbol{\Phi}+\mathbf{Q}_{\Phi} \dot{\boldsymbol{\Phi}}$. Then the similarly as in (2) manifold can be selected as

$S_{\Phi}=\left\{(\mathbf{q}, \dot{\mathbf{q}}): \mathbf{C}_{\Phi} \mathbf{\Phi}+\mathbf{Q}_{\Phi} \dot{\boldsymbol{\Phi}}-\xi_{\Phi}^{r e f}(t)=\boldsymbol{\sigma}_{\Phi}=0\right\}:$

Assuming that $\mathbf{Q}_{\Phi}^{-1}$ exists, the control input enforcing convergence to the manifold (12) can be determined as

$\mathbf{F}_{\Phi}^{*}=\operatorname{sat}\left(\mathbf{F}_{\Phi e q}-\mathbf{Q}_{\Phi}^{-1} \mathbf{\Psi}\left(\boldsymbol{\sigma}_{\Phi}\right)\right)$

$\mathbf{F}_{\Phi e q}=\mathbf{d}_{\Phi}-\mathbf{Q}_{\Phi}^{-1}\left(\mathbf{C}_{\Phi} \dot{\boldsymbol{\Phi}}-\dot{\xi}_{\Phi}^{r e f}(t)\right)$

Inverse transformation $\mathbf{F}=\left(\mathbf{J}_{\Phi} \mathbf{M}^{-1} \mathbf{B}\right)^{-1} \mathbf{F}_{\Phi}^{*}$ gives control in the original state space.

For verification of the proposed approach the experimental system consisting of: two $400 \mathrm{~W} 3$-phase Maxon brushless motors $\left(J=831 \mathrm{~g} / \mathrm{cm}^{2}, K_{T}=85 \mathrm{mNm} / \mathrm{A}\right.$, Maxon 4-Q-EC servo amplifier DES 70/10) in current regulation mode; $10.000 \mathrm{ppr}$ encoders; a dSPACE ${ }^{\circ} 1103$ real-time controller with 100 $\mu \mathrm{sec}$ measurement sampling rate and a $1 \mathrm{msec}$ control output sampling rate in bilateral systems configuration is used. Structure of the overall system is depicted in Fig. 1. In order to make contact with different environment the obstacles are put on the right side - hard - steal rode, and on the left side - a sponge. This way experiments related to contact with very different environment are available. Results depicted in Fig. 2., shows that system controller is capable of handling both, contact with soft and contact with hard environments on the slave side. The proposed structure guaranties the functional relation between master and slave system (equality of positions and forces) but it does not influence amplitude for any of these variables.

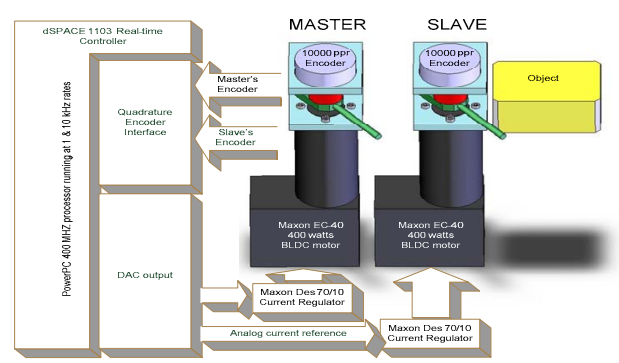

Fig 1. Experimental system for bilateral operation

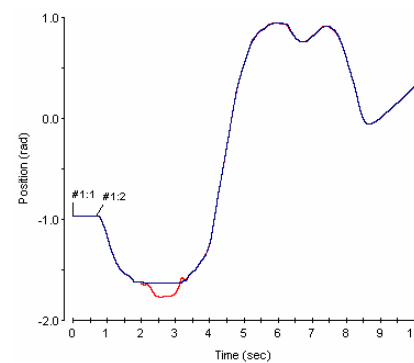

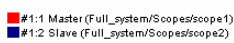

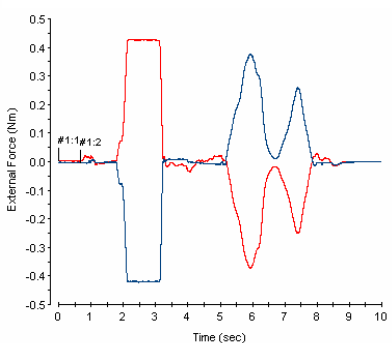

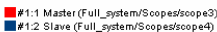

Fig. 2 Transients in bilateral control system (a) position response of master and slave sides, (b) forces of master and slave side

\section{CONCLUSIONS}

In this paper it has been shown that the same approach can be used in controlling mechanical systems in interaction or for establishing desired functional relation between systems thus providing the same framework to bilateral and "function control" systems. The structure of the controller is selected to fulfill Lyapunov stability criteria and enforce the stability of the motion on the selected manifold. Experiments on bilateral control system are shown confirming all theoretical predictions.

\section{ACKNOWLEDGEMENT}

The part of this research is done within TÜBİTAK Project 104E021.

\section{REFERENCES}

Onal, C. D. Sabanovic, A. (2005), Bilateral Control with a Reflex Mechanism on the Slave Side", Proc. of the 31st Annual Conf. of the IEEE Industrial Electronics Society (IECON2005), pp. 195200

Onal, C. D., (2005), Bilateral Control-SMC approach, MSc. Thsis, Sabanci University, Istanbul

Drazenovic B., (1969) The invariance conditions in variable structure systems,_Automatica, vol.5, pp. 287-295, Pergamon Press

Katsura, S. and Ohishi, K, (2007) Modal System Design of Multirobot System by Interaction Mode Control, IEEE Tran on IE, Vol. 54, No. 3, pp. 1537-1546

Strassberg, Y.; Goldenberg, A.A.; Mills, J.K., 1992, A New Control Scheme For Bilateral Teleoperating Systems: Performance Evaluation And Comparison," Intelligent Robots and Systems, 1992., Proceedings of the 1992 IEEE/RSJ International Conference on , vol.2, no.pp.865-872, 7-10 Jul

T. Tsuji, K. Ohnishi, (2005), A Controller Design Method of Decentralized Control System, IEEJ Int. Power Electronics Conf. (IPEC-NIIGATA).

T. Tsuji, K. Ohnishi, (2005), A Controller Design Method of Decentralized Control System, IEEJ Int. Power Electronics Conf. (IPEC-NIIGATA).

Toshiaki Tsuji, Kouhei Ohnishi Asif Sabanovic (2006), A Controller Design Method Based on Functionality" In the Proc. Of $9^{\text {th }}$ IEEE International Workshop on Advanced Motion Control, pp. 171176, Istanbul March 27-29

Tsuji, T. , (2005) Motion Control For Adaptation To Human Environment, Ph.D. Dissertation, Keio University, Japan,

V. Utkin, J. Guldner and J. Shi, (1999), Sliding Modes in Electromechanical Systems, Taylor \& Francis 